\begin{tabular}{|c|c|c|c|c|c|}
\hline Revista Clio América & ISSN: 1909-941X & Vol. 11 & No. 21 & enero - junio de 2017 & 88 - 107 \\
\hline \multicolumn{6}{|c|}{ DOI: http://dx.doi.org/10.21676/23897848.2079 } \\
\hline
\end{tabular}

\title{
La innovación y educación: Variables claves para la competitividad de las empresas
}

\author{
Innovation and education: Key variables for the competitiveness of enterprises
}

RESUMEN: Este artículo es una reflexión sobre la educación como el motor de desarrollo económico, social y humano de las regiones. Se centra en Colombia y sostiene que no se puede hablar de competitividad sin esta crucial variable que se traduce en prosperidad y progreso. La educación y la competitividad son aliadas inseparables e interdependientes. La innovación es necesaria para mejorar la competitividad porque así las empresas ofrecen productos o servicios únicos que reducen su competencia, a la que puede incluso eliminar, si su propuesta es diferenciadora para aquellos consumidores que siempre buscan innovaciones en los productos y si los satisfacen y superan sus expectativas. La innovación debe ser vista como un aliado fuerte para las compañías de vanguardia que sean seguidas por otras firmas, en tanto modelos de innovación.

Palabras clave: educación; innovación; competitividad; investigación; Colombia.

JEL: M10, M14, M15, M16

ABSTRACT: This article is a reflection about education as the motor of the economic, social and human development of territories. It centers around Colombia. The main argument is that competitiveness does not exist unless Education, a crucial variable that translates into prosperity and progress, is taken into account. Education and competitiveness are thus interdependent inseparable allies. Companies need to innovate in order to be competitive, because once they offer unique products or services they can reduce or even eliminate competition -a distinctive proposal gets positioned among customers looking for product or service innovation if they get satisfied and if their expectations are met. Companies wanting to be at the head in their field, should see innovation as a dynamic partner, one that would guarantee their becoming a model for other companies to follow.

Keywords: education; innovation; competitiveness; research; Colombia.

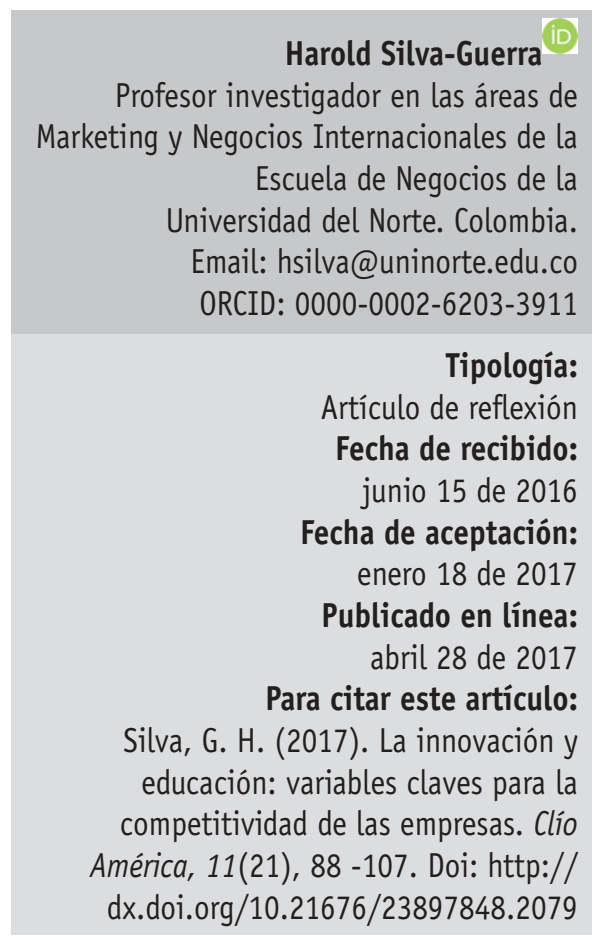




\section{INTRODUCCIÓN}

Actualmente el escenario global esta signado por la globalización, la cual ha cambiado todos los procesos económicos, culturales, sociales y tecnológicos a lo largo y ancho del mundo; es por eso que se hace necesario generar un cambio drástico en los procesos de educación, y por supuesto, en innovación en todas las naciones para convertirse en países con muchas oportunidades comerciales. Todo lo anterior, sería un gran viaje para lograr altísimos procesos de competitividad que se consiguen aprovechando los recursos humanos, naturales, geográficos, sociales, económicos y tecnológicos de las naciones, haciendo énfasis en Colombia.

Muchas naciones del mundo se han transformado en verdaderos ejemplos, los cuales se han basado en la educación como motor trascendental para ser realmente competitivos, pero esto solo se logró con el trabajo mancomunado de muchos actores, trabajar desorganizadamente y como islas es sinónimo de atraso, lo que hoy se busca es el trabajo coherente del Estado, las empresas y los trabajadores, si esta sinergia no ocurre, seguiremos observando naciones ricas, pobres y en miseria absoluta. Hoy solo debemos hablar de lograr altas tasas de competitividad y de hacer lo que realmente debemos hacer, progresar y transformar.

\section{Definición y reflexión sobre el concepto de competitividad}

Antes de abordar esta temática podemos identificar varios conceptos de autores y organismos para contextualizar nuestra discusión sobre la educación y la innovación:

a) World Economic Forum, 2016: define competitividad como el conjunto de instituciones, políticas y factores que determinan el nivel de productividad de un país.

b)Institute for Management Development - IMD, 2016: habilidad de las naciones para crear y mantener un ambiente en el cual puedan competir las empresas.

c) Consejo Privado de Competitividad, 2015: se define como productividad y referencia a la eficiencia con que una nación maneja sus recursos humanos, naturales, financieros, entre otros utilizables en la producción de sus bienes y servicios.

d) Comisión Presidencial Norteamericana sobre Competitividad Industrial, 1985: es la organización de una nación para sostener y difundir su inclusión en los negocios globales y aumentar paralelamente el nivel de la calidad de vida de sus asociados.

e) Garelli, 2006: ilustra el proceso evolutivo del concepto de competitividad y analiza el desarrollo a través de pensadores de los últimos tres siglos (Ricardo, Weber, Schumpeter, Sloan, Drucker, Solow) como un proceso de agregación que concluye en la propuesta del profesor Michael Porter.

f) Porter, 1991: es la productividad en la que un país, región o clúster usa sus recursos humanos y naturales.

g)Ara0z, 1998: la competitividad es la consecuencia del entretejido de una cadena de variables económicas, geográficas, sociales y políticas que hacen parte de la base estructural del desarrollo de un país.

h)Krugman, 1994: critica la expresión y lo considera como una obsesión peligrosa cuando se utiliza a los países.

i) Romo y Musik (2005, p.1): renovó la discusión y continuó usando esta definición sin tener en cuenta que su reiterado manejo, sin ningún control, puede causar distorsión en su significado.

j) Reinert (1995, p.21): continuamente mal utilizado y mal definido, la palabra competitividad, usado comedidamente, describe una particularidad significativa de la economía mundial. Este concepto araña la superficie de asuntos trascendentales que son centrales para entender la distribución de la riqueza, tanto nacional como internacional. El vocablo competitividad apunta hacia cuestiones que han sido centrales en lo público por lo menos durante los últimos 500 años aunque bajo desiguales coberturas. Asi mismo, sustentaré que el término competitividad, usado adecuadamente, revela debilidades significativas en el arquetipo de la economía neo-clásica.

k) Otero $(2006$, p. 10): que esta se discute de forma habitual, ya sea en el escenario universitario 0 público, aunque se le asigna gran importancia, puesto que influye notoriamente sobre el crecimiento de las empresas o de las economías de los países, su preponderancia no está suficientemente 
definida y concretada, pues hay numerosas variables relacionadas con esta designación.

l) Scott y Lodge (1985): la habilidad de una nación de establecer, manufacturar, comerciar y/o utilizar productos en el comercio global al mismo tiempo que gana retornos sustanciales sobre sus recursos.

m) Haguenauer (1989): la empresa debe generar recursos con modelos concretos, solicitados por los diferentes compradores pero siempre privilegiando su producción ya sea, rebajando el tiempo de producción, o maximizando los procesos industriales.
n)Wasserman (2016): la competitividad depende de la productividad del trabajo, de la educación, de la fortaleza de la moneda, del acceso a mercados y de mil factores más, no solo de la ciencia. Hay resultados de la ciencia que potencialmente mejoran la competitividad, la mayoría tienen poco que ver con ella (Fundación Alemana para la Investigación - DFG).

Presentados diferentes puntos de vista relacionados a la competitividad, se muestra la Tabla 1 para contrastar los términos más relevantes:

\section{Tabla 1.}

Matriz nomotética de términos relevantes de competitividad

\begin{tabular}{|c|c|}
\hline AUTOR & TÉRMINOS RELEVANTES \\
\hline World Economic Forum, 2016 & Productividad \\
\hline Institute for Management Development - IMD, 2016 & Ambiente, competencia \\
\hline Consejo Privado de Competitividad, 2015 & Productividad, eficiencia, recursos \\
\hline $\begin{array}{l}\text { Comisión Presidencial Norteamericana sobre } \\
\text { Competitividad Industrial, } 1985\end{array}$ & $\begin{array}{l}\text { Participación, mercados internacionales, calidad de } \\
\text { la población }\end{array}$ \\
\hline Garelli, 2006 & Proceso evolutivo del concepto de competitividad \\
\hline Porter, 1991 & Productividad, recursos humanos y naturales \\
\hline Araoz, 1998 & $\begin{array}{l}\text { Factores económicos, geográficos, sociales y } \\
\text { políticos }\end{array}$ \\
\hline Krugman, 1994 & Obsesión peligrosa \\
\hline Romo y Musik (2005) & Índices de competitividad nacional, globalización \\
\hline Reinert (1995) & $\begin{array}{l}\text { Característica importante de la economía mundial, } \\
\text { distribución de la riqueza }\end{array}$ \\
\hline Otero (2006) & Crecimiento de las naciones \\
\hline Scott y Lodge (1985) & Crecimiento de los países \\
\hline Haguenauer (1989) & $\begin{array}{l}\text { Producción de bienes con patrones de calidad } \\
\text { particulares }\end{array}$ \\
\hline Wasserman (2016) & Dependencia de la competitividad, ciencia. \\
\hline
\end{tabular}

Fuente: Elaboración propia 


\section{Educación y competitividad en Colombia}

La economía de Colombia marcha por buen camino, con un desarrollo pausado pero convincente, así lo señalan los resultados del Departamento Administrativo Nacional de Estadística (DANE). Para el 2015 la economía colombiana creció 3,1 \% y se pronostica un $3 \%$ de crecimiento para el 2016.

Alcanzar que las Pymes de Colombia empiecen cada vez más a buscar otros mercados para su portafolio. Esto constituye una labor por realizar. El Gobierno Nacional con los tratados de libre comercio suscritos, convirtió en un proyecto nacional optimizar la competitividad de las compañías y la más óptima infraestructura para promocionar el comercio global. Así las cosas, la región Caribe colombiana está en la mira de muchos empresarios por su excelente ubicación estratégica para facilitar la logística comercial. Asimismo, se han ejecutado inversiones para desarrollar demandas en conectividad, infraestructura e inversión social, por ejemplo, la recuperación y la navegabilidad del río Magdalena, con lo cual se busca disminuir los costos del transporte entre la costa Atlántica y el interior del país.

Otras obras de importancia capital es el arreglo de las carreteras entre las principales ciudades de la costa colombiana, como también las entradas que favorecerán y agilizaran el flujo vehicular. Lo ideal sería con todas las capitales de la región Caribe.

La región Caribe tiene tres puertos substanciales (Barranquilla, Santa Marta y Cartagena) y una entrada al río, lo cual ha mejorado sectores como el turístico, agroindustria, petroquímico, manufacturero, farmacéutico, metalmecánico, dotación para hospitales, moda, entre otros. Es así que para desarrollar la competitividad se debe trabajar simultáneamente todas las ciudades de la zona.

Colombia se mantuvo en el puesto 61 entre 138 economías del ranking mundial de competitividad del Foro Económico Mundial en 2016, los beneficios en crecimiento y desarrollo no son importantes. Sin duda que todas las inversiones públicas y privadas, cambiaran y dinamizaran la progresión de la zona, pero para conseguir el triunfo la educación y la innovación serán trascendentales.

La educación es el motor de desarrollo económico, social y humano de las regiones; no se puede hablar de competitividad sin esta crucial variable que traduce en prosperidad y progreso, es importante volverla obligatoria, tanto en primaria secundaria. No podemos darle ventaja al analfabetismo exponencial que se vemos a diario en la calles con niños pidiendo dinero, los cuales deben estar en una escuela preparándose para su vida. Lo anterior se traduce en una miseria mental que mata el espíritu de las personas. Sin educación no se puede concebir la competitividad ni hablar de desarrollo a todos los niveles. Es urgente aumentar los cupos en las universidades tanto públicas como privadas y por qué no, en un futuro volverla obligatoria. Es necesario que las personas conozcan un oficio y se vuelvan expertos para llegar a la innovación y alcanzar niveles de competitividad.

En este orden de ideas, una extraordinaria infraestructura en la educación es el deber ser para que los estudiantes no se distraigan en cosas pequeñas como por ejemplo; una silla no confortable, un tubo mal colocado en el aula, ruidos, docentes flexibles en los horarios porque si el horario se reduce la temática también, un minucioso seguimiento a la catedra para que se cumpla al $100 \%$, profesores con vocación para enseñar (no es hacerlo por hacerlo, se debe medirlos y desarrollar una herramienta), los docentes deben generar debate al interior de las clases, no todo está en Google, los profesores deben profundizar al interior de la catedra y la temática, ir a lo más profundo de lo que se está enseñando. ¿Para qué sirve el conocimiento? ¿Me prepara para tomar decisiones? ¿Qué tanto me sirve el conocimiento? Lo importante no es lo tengo sino que desarrollo con lo tengo.

Todas las personas pueden mejorar a través del tiempo muchos factores como lo social que deriva en el mejor comportamiento de los individuos y aprenden diversas cosas como el conocimiento, costumbres, valores y formas de comportarse, con el objetivo de ser mucho más como humano y dar lo mejor de sí 
para la sociedad, y por supuesto, para las empresas; es aquí donde la educación cobra muchísima importancia en la transformación de las personas para ayudar a ser multiplicadores con la premisa que la educación cambia, transforma y motiva, siempre y cuando, para que esta tenga éxito debe ser equitativa e imparcial donde todas las personas tengan iguales oportunidades y el nivel de competencia se desarrolle en la sociedad en iguales condiciones, la educación es un deber y un derecho para el progreso de los pueblos.

\section{Para León (2007)}

los hombres requieren aprender lo que no le es natural, lo que natura no le ha dado y desarrollar lo que se le ha proporcionado por consanguinidad. Por tal razón necesita de otros y la cultura para afianzar su camino por la tierra. Lo anterior es el proceso de la educación (p. 596).

La educación y la competitividad son aliadas inseparables donde cada una de las partes se necesita de manera paralela.

\section{Según el Informe Nacional de Competitividad} 2014-2015:

Los desafíos de Colombia en cuanto a los niveles de su formación primaria y media no son mínimos. Alrededor del $40 \%$ de los estudiantes de último año inscritos en educación superior están en los niveles más bajos de las pruebas Saber 11, y la escasa proporción (18\%) de quienes califican en un lugar descollado, es muy bajo. En la última prueba PISA (Programme for International Student Assessment) (2012) los resultados fueron para nada satisfactorios. Más allá de que el país ocupe los últimos lugares frente a los de las naciones de la Organización para la Cooperación y el Desarrollo Económico (OCDE), inquietan el alto porcentaje de alumnos que registra por debajo del nivel 2 en cada prueba y la bajísima proporción de aquellos que califican en los mejores niveles (pp. 23-25).

Especialistas nacionales e internacionales, incluyendo la Organización para la Cooperación y Desarrollo
Económicos $(0 \mathrm{CDE})$, concuerdan en que la calidad de un régimen educativo estriba especialmente de la calidad de sus docentes. "Falta mucho por hacer para optimizar la valoración social de la docencia y aumentar el estatus socio-económico de estos" (Informe Nacional de Competitividad 2014-2015, p.25).

Estimular el desarrollo de la educación terciaria actual y pensar otras opciones para la transformación, técnica y tecnológica, de acuerdo a las necesidades que exija la nueva realidad económica (Silva, 2014). El 8,5\% de las carreras de Colombia están acreditados en alta calidad y la totalidad de estos se agrupa en los programas de pregrado (Informe Nacional de Competitividad 2014-2015, p.26-27).

\section{Para Moreno-Brid y Ruiz-Nápoles (2009):}

Robustecer en Latinoamérica las universidades y la investigación, fundamentalmente las estatales, es un elemento importante para desarrollar la competitividad global de su estructura productiva y acceder a un nivel de alta expansión económica de largo plazo. Infaliblemente, en la zona las instituciones de educación públicas son las que mantienen el progreso de la ciencia y la tecnología; sin este conocimiento sería altamente imposible que la zona obtuviera el desarrollo económico. ¿Cuál es la huella económica de las instituciones de educación superior estatales? ¿Cuáles son los canales por los que las universidades e instituciones de investigación públicas estimulan la innovación tecnológica en Latinoamérica? ¿Cómo es que estos canales pueden hacerse más eficaces para promover el desarrollo económico? ¿Cuáles son las primordiales dificultades? (p.8).

\section{Innovación y competitividad}

La innovación es necesaria para mejorar la competitividad porque así las empresas ofrecen productos o servicios únicos donde su competencia se reduce porque las puede eliminar debido que su propuesta es diferenciadora para aquellos consumidores que siempre buscan nuevas innovaciones en los productos y satisfacen y superan sus expectativas; la innovación debe ser vista como un aliado fuerte para 
que las compañías sean consideradas de vanguardia, y sean seguidas por otras firmas y ser modelos de innovación.

La innovación es un proceso que supone un cambio 0 una mejora a la idea original, proceso, producto 0 servicio cuyo objetivo es producir cambios positivos (CEIM, 2001).

Para la CEPAL - Naciones Unidas (2004) exponen los siguientes puntos en torno a políticas para impulsar la innovación y el desarrollo tecnológico:

a) El desarrollo obedece a una suma de variables productivas y de incorporación de conocimiento a la producción, es decir la innovación), por ejemplo, con capacitación de la mano de obra en la gestión de las compañías.

b) La velocidad de la innovación establece el camino por el cual la prosperidad de una nación se aproxima o se aleja del dominante en las áreas más perfeccionadas del globo, que pueden considerarse en las fronteras tecnológica internacional.

c) La innovación y el conocimiento simbolizan las voluntades de las compañías por comprometerse en la producción y la gestión, de nuevas tecnologías de fabricación y gestión, e ir corrigiendo progresivamente en el futuro cercano implementos novedosos para la tecnología.

d) La economía global registró en los últimos años un considerable crecimiento en el avance de la tecnología, lo cual estimula la aparición constante de nuevas actividades permitiendo manufacturar, comerciar y adquirir bienes, servicios y conocimientos, entre otros.

e) Los avances técnicos, como consecuencia de la innovación en las diferentes fases de la fabricación es un proceso holista en el cual tienen participación factores que intervienen en el sistema nacional de innovación.

f) El desarrollo productivo de las economías que se formulan para disminuir el espacio entre la producción con las más adelantadas tecnologías requiere la aceptación de políticas públicas reservadas a establecer y perfeccionar habilidades de innovación que faciliten usar las ventajas de la rapidez del cambio tecnológico y la apertura económica.

g) Es muy importante instituir un ambiente más apropiado a los esfuerzos de organizar verdaderas capacidades de cambio (p. 211-213).

En suma, las compañías están en la obligación de montar políticas internas de competitividad para volver a definir los procesos desde diferentes ópticas, teniendo en cuenta la premisa de presentar un producto 0 un servicio de calidad, en cuanto se refiere a su desempeño, valor final, y lugares donde se puedan obtener o maneras de acceder a cualquiera de los dos (Silva, 2014, p.11).

Para López et al. (2009), “desde el punto de vista empresarial, y con un enfoque macroeconómico, podríamos afirmar que no existen países o regiones competitivos sino empresas competitivas (la riqueza no se crea a nivel macro sino a nivel micro) (p. 128).

\section{Silva (2014) asevera que:}

En esta época de mutua dependencia mundial, los negocios internacionales constituyen la vía más expedita para el desarrollo económico de las naciones. La tesis del comercio internacional marca una simbiosis positiva entre la liberación comercial y el fomento económico. Muchas naciones se especializan en algunos sectores que muestran una ventaja comparativa y así acrecientan la producción total de bienes y servicios, con lo que logran una mejor fuente de recursos en el caso de las economías cerradas (Figura 1). Con la apertura internacional se plantea la tesis de que las variables externas llevan a hacer más competitivo un país, puesto que las firmas poco eficientes la competencia las acabara, y aquellas que sobrevivan están obligadas, para salvarse del naufragio definitivo, a implementar desde la gerencia políticas de corte internacional y propender por altísimos niveles de capacidad administrativa para sostenerse en el mercado (pp.13-14). 


\section{Figura 1.}

\section{Retos de la competitividad}

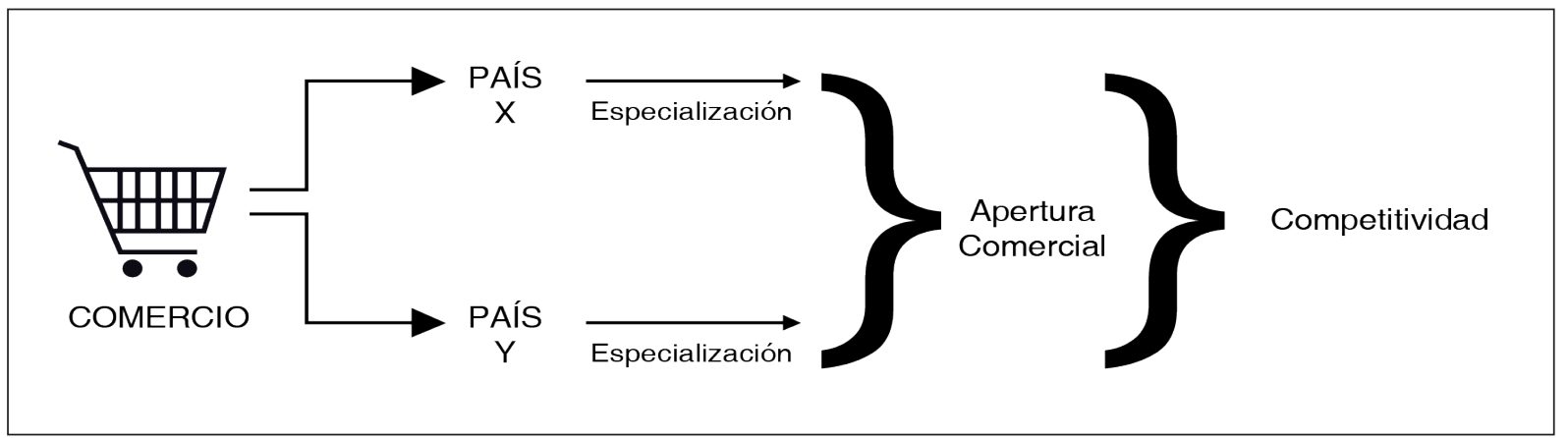

Fuente: Silva, 2014, p.15

En suma, la renovación constante que exigen los mercados globales, las novedades de la competencia empresarial, y hasta las modas publicitarias sobre el uso de la tecnología que llegan a los medios de comunicación, convergen en una innovación tecnológica permanente (López, et al, 2009, p.131).

\section{El conocimiento: un aliado de la competitividad}

Actualmente no se duda que el conocimiento es una ventaja competitiva significativa. Coexiste una correspondencia directa entre la educación y la productividad, es por ello, que la calidad del capital humano es primordial, no solo para que las compañías progresen, sino de igual forma para el desarrollo de los países.

Para convertirnos en naciones más competitivas lo primero que se debe realizar es organizarnos internamente, y por supuesto, prepararse y proyectarse. Una variable fundamental es que las firmas conozcan sobre lo que hacen, eso lleva a la necesidad invariable de capacitarse académicamente. La educación es importante para estudiar los mercados foráneos, para conocer a los clientes y para enfrentar la competencia. En ese sentido la universidad debe verse como un aliado.

La capacitación laboral es fundamental en el exitoso desempeño internacional y la formación del personal es una inversión que a largo plazo fortalece el sector productivo. Por ejemplo, algunos países han insistido en la educación como el principal soporte e impulso de su desarrollo. Casos como el de Corea del Sur, que 60 años atrás, era un país pobre y atrasado comparado con el nuestro, ahora es una verdadera potencia en todos los órdenes. Su base principal fue la educación y con esta lograron convertir a muchos coreanos en excelentes profesionales con maestrías y doctorados de las mejores universidades del mundo. Taiwán pasó de ser una sociedad de agricultores a una industrial, con el mismo esquema. Si ellos pudieron por qué nosotros no.

Corea del Sur a través de los años en las pruebas PISA, se perfila como una nación con excelentes resultados en matemáticas, lectura y en competencia científica. Es la comprobación de un milagro en educación, en 60 años no solo ha permitido a la nación salir del analfabetismo desde su independencia en el año 45 hasta ubicarla en lo más alto de la OCDE (Arrizabalaga, 2013).

Arrizabalaga (2013) afirmó que:

El fuerte empeño de educación de los coreanos no se observa en ninguna otra parte del orbe. El entusiasmo de los coreanos por la educación es asombroso. Una nación de campesinos analfabetos y semianalfabetos con solo una muy pequeña clase urbana se ha evolucionado en una de los estados con mejor educación de la tierra. A través de la educación se reformó esta nación posteriormente de la guerra social y económicamente. El principio esencial de todo el sistema educativo coreano es im- 
pulsar la formación como medio para el crecimiento económico del país. El nacionalismo es muy hondo y la voluntad de los alumnos de apoyar al desarrollo de su país está muy desarrollada (p. 1).

Casi el ciento por ciento de sus educandos finaliza la secundaria y aproximadamente el $60 \%$ recibe un diploma universitario. $Y$ todo esto es fortalecido con 4 ó 5 horas de clase privadas, más un período de estudio en su hogar, complementando con un aprendizaje en bibliotecas especializadas para jóvenes de secundaria. Los surcoreanos estudian 16 horas más semanalmente que la media de la OCDE. Corea del Sur destina el $7 \%$ de su Producto Interno Bruto en la educación con el fin que su juventud tenga acceso a las mejores universidades de los países desarrollados en todo el mundo. Con esto han logrado formar una clase empresarial muy poderosa que se encuentra diseminada por todo el planeta (Arrizabalaga, 2013).

Para Oppenheimer (2009):

El modelo de educación de Singapur tuvo la visión de cambiar a esta nación en un país angloparlante con educación bilingüe, donde los alumnos aprenden inglés como primera lengua y su idioma materno (mandarín, tamil o malayo) como segundo. Eso favoreció a convertir a Singapur en un centro significativo del comercio mundial. Igualmente convirtió el sistema educativo en una de las más fuertes meritocracias del globo, que produce empleados altamente distinguidos y que exporta cada vez más productos de alta tecnología. La meritocracia académica de esta nación principia en primera medida, donde los niños son distribuidos en un ranking acorde a su desempeño académico, desde el primero hasta el último (p.1).

En Japón, el sistema de educación podría ser muy confuso, no obstante, en los aspectos más fundamentales exhibe muchas semejanzas con el que pueda existir en su país. La universidad empieza después de doce años de estudios secundarios, más seis años de formación básica y seis años de estudios secundarios divididos en inferior ( 3 años) y superior (3 años) (Universia, 2015).

Por otro lado, en Finlandia para los primeros seis años de la primaria los niños tienen en casi todos los cursos el mismo profesor, éste tiene como responsabilidad que ningún estudiante quede por fuera. Lo anterior es un modo de vigorizar su seguridad emocional. La gratuidad comprende desde el preescolar hasta la universidad (Arrizabalaga, 2013).

\section{Arrizabalaga (2013) asevera que:}

La victoria finlandesa obedece a que articulan tres niveles fundamentales: la familia, la escuela y los recursos socioculturales. Estas tres variables van ligadas y trabajan de manera ordenada. Los papas tienen la certeza que son los primeros comprometidos de la educación de sus hijos, por encima de la escuela y mejoran el esfuerzo que se hace en el colegio. En este país el $80 \%$ de las familias visitan la biblioteca los fines de semana. Una gran diferencia es la alta cualificación académica de los maestros en Finlandia, especialmente en educación básica. Los mejores profesores se ubican en los primeros años de instrucción (p.1).

La educación suiza es gratuita y obligatoria. Todos los estudiantes (niños y niñas) deben ir a la escuela, así como los inmigrantes. Habitualmente la educación obligatoria principia con siete años y dura unos nueve años en total. Numerosos niños y niñas pasan dos años en la guardería antes de iniciar la escuela. La educación suiza es distinguida en todo el globo. Los estudiantes suizos registran los resultados más elevados en habilidades matemáticas y en exámenes de ciencia en paralelo con otros alumnos de Europa (Just Landed, 2015).

El sistema educativo suizo está descentralizado y está operado a tres niveles políticos: federal, cantonal y municipal. El gobierno federal inspecciona la educación de manera nacional y es garante de que los niños asistan a la escuela en general. A causa de la variedad de culturas e idiomas, cada cantón fiscaliza su sistema educativo. Los colegios enseñan en alemán, francés, italiano o romance, esto depende del cantón donde estén localizados (Just Landed, 2015).

La escolarización es obligatoria en Inglaterra para todos los niños entre 5 y 16 años de vida. El sistema educativo de este país imperante en la actualidad, inició en 1988 años en el cual se implantó la nueva 
norma educativa, Education Reform Act 1988, que forma la base de su sistema educativo y que ha tenido algunos cambios en los últimos 20 años, principalmente a causa de los cambios políticos. Con esta, y sus modificaciones, se implantaron variables muy trascendentales en el sistema educativo inglés entre los que cabe subrayar la aparición del nacional currículum, nuevos métodos para evaluar el progreso de los estudiantes, para acrecentar la eficiencia de las escuelas basadas en desarrollar la competitividad, nuevos métodos de financiación que facilitan más poder y autonomía a las escuelas, y programas de cooperación y asistencia entre ellas (Universia, 2015).

En Francia está instaurado en tres grandes fases: escuela, colegio e instituto. La educación en primaria y secundaria es gratuita, imparcial, laica e imperativa desde los 6 a 16 años. Empero, existen escuelas privadas no sometidas a estos compromisos y particularidades del sistema escolar de esta nación (France.fr, 2015).

En Costa Rica la educación primaria y general básica son obligatorios. Estas y la educación diversificada, son costeadas ciento por ciento por el Estado. El Ministerio de Educación Pública se encarga de este control. Costa Rica registra uno de los niveles educativos más altos de Latinoamérica y es sin lugar a dudas el mejor de Centroamérica. En Chile la educación básica es obligatoria y gratuita, buscando con ello, asegurar el ingreso de toda su población. En Uruguay la educación es gratuita y obligatoria desde preescolar hasta la universidad, se considera que su educación es una de las más adelantadas y progresistas de Latinoamérica. En Colombia la educación en las instituciones públicas es gratuita.

En Colombia, el gobierno del presidente Juan Manuel Santos lanzó en el 2014 el programa educativo llamado "Ser pilo paga", el cual otorga becas a los mejores estudiantes de escuelas públicas para estudiar carreras profesionales en universidades acreditadas por el Ministerio de Educación Nacional. Este programa les cubre el pago de la matrícula y un subsidio de sostenimiento para vivienda y otros. En este orden, el Gobierno colombiano cubrirá el 100 por ciento a los estudiantes que estén interesados en la carrera para ser profesores con becas condonables bajo la modalidad de estudiar dos carreras, una licenciatura y la otra en ciencias básicas o una maestría.

De los anteriores modelos de educación, se presenta la Tabla 2 para ilustrar las características más relevantes:

\section{Tabla 2.}

\section{Modelos de educación}

\begin{tabular}{|c|c|c|c|}
\hline PAÍs & $\begin{array}{l}\text { CARACTERÍSTICA MÁS } \\
\text { RELEVANTES }\end{array}$ & ENSEÑANZA & ETAPAS ESCOLARES \\
\hline $\begin{array}{l}\text { Corea del } \\
\text { Sur }\end{array}$ & $\begin{array}{l}\text { - Excelentes pruebas PISA } \\
\text { - Pasión por la educación } \\
\text { - A través de la educación } \\
\text { se reconstruyo el país } \\
\text { - } 7 \text { \% del PIB es dedicado } \\
\text { a la educación } \\
\text { - Educación = inversión }\end{array}$ & $\begin{array}{l}\text { - Obligatoria } \\
\text { - Se puede cursar en } \\
\text { la educación pública } \\
\text { aunque no hay dife- } \\
\text { rencia entre cursarla } \\
\text { en un tipo de centro } \\
\text { privado o público }\end{array}$ & $\begin{array}{l}\text { Escuela elemental (con } \\
\text { una duración de seis } \\
\text { años), Primaria (perio- } \\
\text { do de tres años), high } \\
\text { school o institutos (tres } \\
\text { años), junior college (dos } \\
\text { o tres años) y universida- } \\
\text { des ( } 4 \text { años) }\end{array}$ \\
\hline Singapur & $\begin{array}{l}\text { - Se convirtió en una na- } \\
\text { ción bilingüe } \\
\text { - Sistema educativo fuerte } \\
\text { (meritocracia) }\end{array}$ & $\begin{array}{l}\text { - Gratuita, todos los } \\
\text { colegios son públi- } \\
\text { cos y financiados } \\
\text { por el Estado }\end{array}$ & $\begin{array}{l}\text { - Grado } 1 \text { a } 12 \\
\text { - Preescolar (4 años). } \\
\text { - Primary School (6 años) } \\
\text { - Secondary education }\end{array}$ \\
\hline
\end{tabular}




\begin{tabular}{|c|c|c|c|}
\hline PAÍS & $\begin{array}{l}\text { CARACTERÍSTICA MÁS } \\
\text { RELEVANTES }\end{array}$ & ENSEÑANZA & ETAPAS ESCOLARES \\
\hline Japón & $\begin{array}{l}\text { - Sociedad basada en la } \\
\text { titulocracia }\end{array}$ & $\begin{array}{l}\text { - Educación obliga- } \\
\text { toria (primaria y } \\
\text { secundaria) }\end{array}$ & $\begin{array}{l}\text { - Doce años de formación } \\
\text { académica } \\
\text { - Seis años de educación } \\
\text { elemental } \\
\text { - Tres años de educación se- } \\
\text { cundaria inferior y tres } \\
\text { años de educación secun- } \\
\text { daria superior }\end{array}$ \\
\hline Finlandia & $\begin{array}{l}\text { Durante los primeros seis } \\
\text { años los niños tienen el } \\
\text { mismo docente } \\
\text { - } 80 \% \text { de las familias visi- } \\
\text { tan la biblioteca los fines } \\
\text { de semana } \\
\text { - Los mejores profesores } \\
\text { los ubican en los prime- } \\
\text { ros años de enseñanza }\end{array}$ & $\begin{array}{l}\text { - Gratuidad desde el } \\
\text { preescolar hasta la } \\
\text { universidad }\end{array}$ & $\begin{array}{l}\text { - Guardería (edad de 6-7 } \\
\text { años) } \\
\text { - Escuela primaria obliga- } \\
\text { toria (edad entre } 7 \text { a } 16 \\
\text { años). } \\
\text { - Educación secundaria } \\
\text { no obligatoria (edad } 16 \\
\text { a } 19 \text { años) } \\
\text { - Formación profesional } \\
\text { (edad } 16 \text { a } 19 \text { años) }\end{array}$ \\
\hline Suiza & $\begin{array}{l}\text { - Los estudiantes tienen } \\
\text { los puntajes más altos en } \\
\text { matemáticas y ciencia }\end{array}$ & $\begin{array}{l}\text { - La educación } \\
\text { es gratuita y } \\
\text { obligatoria }\end{array}$ & $\begin{array}{l}\text { Educación primaria, } \\
\text { secundaria y terciaria }\end{array}$ \\
\hline Inglaterra & $\begin{array}{l}\text { - Antes de los } 5 \text { años y } \\
\text { después de los } 16 \text { años, la } \\
\text { enseñanza no es obligatoria } \\
\text { pero sigue siendo gratuita } \\
\text { hasta los } 18 \text { años } \\
\text { - El sistema educativo actual } \\
\text { depende de leyes promulga- } \\
\text { das por el Parlamento }\end{array}$ & $\begin{array}{l}\text { - Educación obliga- } \\
\text { toria para niños } \\
\text { entre } 5 \text { y } 16 \text { años }\end{array}$ & $\begin{array}{l}\text { - Key Stage } 1 \text { (5 - } 7 \\
\text { años) } \\
\text { - Key Stage } 2 \text { (7 - } 11 \\
\text { años) } \\
\text { - Key Stage } 3 \text { (11 - } 14 \\
\text { años) } \\
\text { - Key Stage (14 - } 16 \\
\text { años) }\end{array}$ \\
\hline Francia & $\begin{array}{l}\text { - Los colegios y centros públi- } \\
\text { cos de enseñanza coexisten, } \\
\text { dentro del servicio público } \\
\text { de enseñanza, con centros } \\
\text { privados concertados }\end{array}$ & $\begin{array}{l}\text { - La educación en } \\
\text { primaria y secun- } \\
\text { daria es gratuita, } \\
\text { neutra, laica e } \\
\text { imperativa de los } 6 \\
\text { a los } 16 \text { años }\end{array}$ & $\begin{array}{l}\text { - Escuela primaria, edu- } \\
\text { cación secundaria y } \\
\text { educación superior }\end{array}$ \\
\hline Costa Rica & $\begin{array}{l}\text { - La educación de Costa } \\
\text { Rica está controlada por el } \\
\text { Ministerio de Educación y es } \\
\text { obligatoria desde primaria } \\
\text { hasta secundaria (con } 16 \\
\text { años) }\end{array}$ & $\begin{array}{l}\text { - La educación prima- } \\
\text { ria y general básica } \\
\text { son obligatorios }\end{array}$ & $\begin{array}{l}\text { - Educación inicial (menores } \\
\text { de } 6 \text { años) } \\
\text { - Educación básica (desde } \\
\text { los } 6 \text { años) } \\
\text { - Educación primaria } \\
\text { - Educación secundaria } \\
\text { - Educación universitaria }\end{array}$ \\
\hline
\end{tabular}




\begin{tabular}{|c|c|c|c|}
\hline PAís & $\begin{array}{l}\text { CARACTERÍSTICA MÁS } \\
\text { RELEVANTES }\end{array}$ & ENSEÑANZA & ETAPAS ESCOLARES \\
\hline Chile & $\begin{array}{l}\text { - Es una de las más avanzadas } \\
\text { y reconocidas de América } \\
\text { Latina } \\
\text { - La tasa de alfabetización } \\
\text { supera el } 96 \%\end{array}$ & $\begin{array}{l}\text { - La educación bási- } \\
\text { ca es obligatoria y } \\
\text { gratuita }\end{array}$ & $\begin{array}{l}\text { - Preescolar (de } 5 \text { a } 6 \text { años) } \\
\text { - Básica (6 a } 13 \text { años) } \\
\text { - Media (14 a } 17 \text { años) } \\
\text { - Superior }\end{array}$ \\
\hline Uruguay & $\begin{array}{l}\text { - Se basa en la gratui- } \\
\text { dad, la laicidad y el libre } \\
\text { acceso a la educación de } \\
\text { todos los ciudadanos }\end{array}$ & $\begin{array}{l}\text { - Educación gratuita } \\
\text { y obligatoria desde } \\
\text { preescolar hasta la } \\
\text { universidad }\end{array}$ & $\begin{array}{l}\text { - Preescolar } \\
\text { - Primaria ( } 6 \text { años) } \\
\text { - Media o secundaria (ciclo } \\
\text { básico ( } 3 \text { años) y segundo } \\
\text { ciclo o bachillerato ( } 3 \\
\text { años) } \\
\text { - Terciaria o superior }\end{array}$ \\
\hline Colombia & $\begin{array}{l}\text { - Programa educativo "Ser } \\
\text { pilo paga" }\end{array}$ & $\begin{array}{l}\text { - La educación en las } \\
\text { instituciones públi- } \\
\text { cas es gratuita }\end{array}$ & $\begin{array}{l}\text { - Educación inicial, la } \\
\text { educación preescolar, la } \\
\text { educación básica (primaria } \\
\text { cinco grados y secundaria } \\
\text { cuatro grados), la educa- } \\
\text { ción media (dos grados y } \\
\text { culmina con el título de } \\
\text { bachiller.), y la educación } \\
\text { superior }\end{array}$ \\
\hline
\end{tabular}

Fuente: Elaboración propia (2016)

La siguiente historia puede tener parecido con la realidad es de una persona con una clara visión sobre el valor de la educación:

Juan Hernández es un joven con muchas aspiraciones en la vida, estudia su bachillerato en una escuela pública de cualquier pueblo latinoamericano, no cancela nada por su educación porque el Estado lo cubre en su totalidad. Su familia es muy pobre, Juan estudia de 7 a.m. a 1 p.m.; en la tarde de 2:30 a 8 p.m. trabaja recogiendo basura para reciclar y vender a empresas recicladoras; es el negocio de su familia. A duras penas les alcanza para comer y pagar el arriendo de la habitación donde viven.

Juan estudia y cumple sus obligaciones académicas de noche, es inquieto y recursivo; estudia con libros prestados de sus compañeros y de la biblioteca de la escuela y estatal. Pasa el tiempo y con muchas dificultades se gradúa del bachillerato, para él los obstáculos fueron su gran motivación. Él desea ser Administrador de Empresas, para ello se presentó en la universidad pública de la región donde habita y se ganó una beca por sus excelentes notas del bachillerato y su notable examen de admisión superando a muchos estudiantes que provienen de escuelas prestigiosas.

Tiene mucha sed de conocimiento y desea ser Administrador de Empresas y se visiona con ser gerente de una compañía. Pasa el tiempo y con muchos problemas y barreras de todo tipo se gradúa como Administrador de Empresas con honores, se gana la medalla de oro por su excelencia académica. 
Después de su grado empieza a laborar en una compañía como asistente de un departamento; pasa el tiempo y se inscribe para concursar por una beca en una universidad anglosajona para una maestría y se la gana aun superando la barrera del idioma (él se preparó con escasos recursos), y la beca le cubre los costos de la matrícula y un tiquete aéreo, no le cobija el alojamiento y alimentación, pero para él no fue un obstáculo. Durante su estancia en el trabajo ahorró y a la vez ayudaba a su familia. Viajó y el dinero que llevaba solo le alcanzaba para un mes. En el país consiguió trabajo de mesero en las horas libres que solo le alcanzaba para cancelar su alojamiento y alimentación. Con su trabajo fuerte y con las ganas de salir adelante se gradúa con todos los honores, la universidad anglosajona le propone realizar un $\mathrm{PhD}$ con beca completa incluyendo su alojamiento y alimentación.

Hoy en día, Juan es presidente de una multinacional con un buen nivel de vida y por supuesto, ayuda a sus hermanos a estudiar. Juan afirma "para mí la educación no solo me transformó, sino a todo mi entorno familiar, gracias por existir educación".

La anterior historia es un vivo ejemplo que la educación transforma al ser humano.

Para la UNESCO (2009) los retos de la educación superior se pueden relacionar de la siguiente manera:

a) Acceso: se debe aumentar el acceso y esta es una gran prioridad para todas las naciones. Sin embargo, el acceso no es suficiente, realmente se debe asegurar el éxito de los alumnos.

b) Equidad: esta debe ser vista con una clara participación de éxito y la finalización de los estudios con apoyo financiero, bienestar para los estudiantes y soportes educativos para alumnos pobres y marginados. c) Calidad: es muy importante que se deba implementar muchos mecanismos para asegurar la calidad y se promueva el acceso y se originen las condiciones para la finalización de los estudios.

d)Internacionalización: todas las universidades deben aumentar la transferencia de conocimiento principalmente hacia naciones en desarrollo e impulsar la circulación de profesionales y aminorar la fuga de cerebros.

e) Regionalización: reconocimiento de los títulos, innovación, investigación, calidad y gobernanza, para lo anterior es imperioso una buena colaboración regional.

f) Globalización: es necesario instaurar sistemas propios de acreditación, trabajo en redes y calidad.

g)Aprendizaje: la liberta académica es trascendental porque se caracteriza por evolución permanente y su volatilidad.

h)Innovación e Investigación: se deben encontrar mecanismos para el financiamiento y el desarrollo de la investigación e innovación.

Los docentes del siglo XXI para estudiantes del siglo XXI deben ser globales con una preparación académica alta con maestrías científicas, doctorados y postdoctorados (Figura 2), son el ADN y eje fundamental para promover, motivar y generar la cultura de la investigación para generar conocimiento y la innovación en las naciones, y por supuesto, en las empresas. Se debe crear una política de cambio a este nivel. Y lo anteriormente expuesto, no es solo para docentes sino para el empresario o profesional, se debe preparar en altos niveles para poder afrontar el nuevo mundo de los negocios que cada día se hace más estrecho y con particularidades que solo se identifican cuando se mezcla la experiencia con la educación. 


\section{Figura 2.}

\section{Investigación e Innovación}

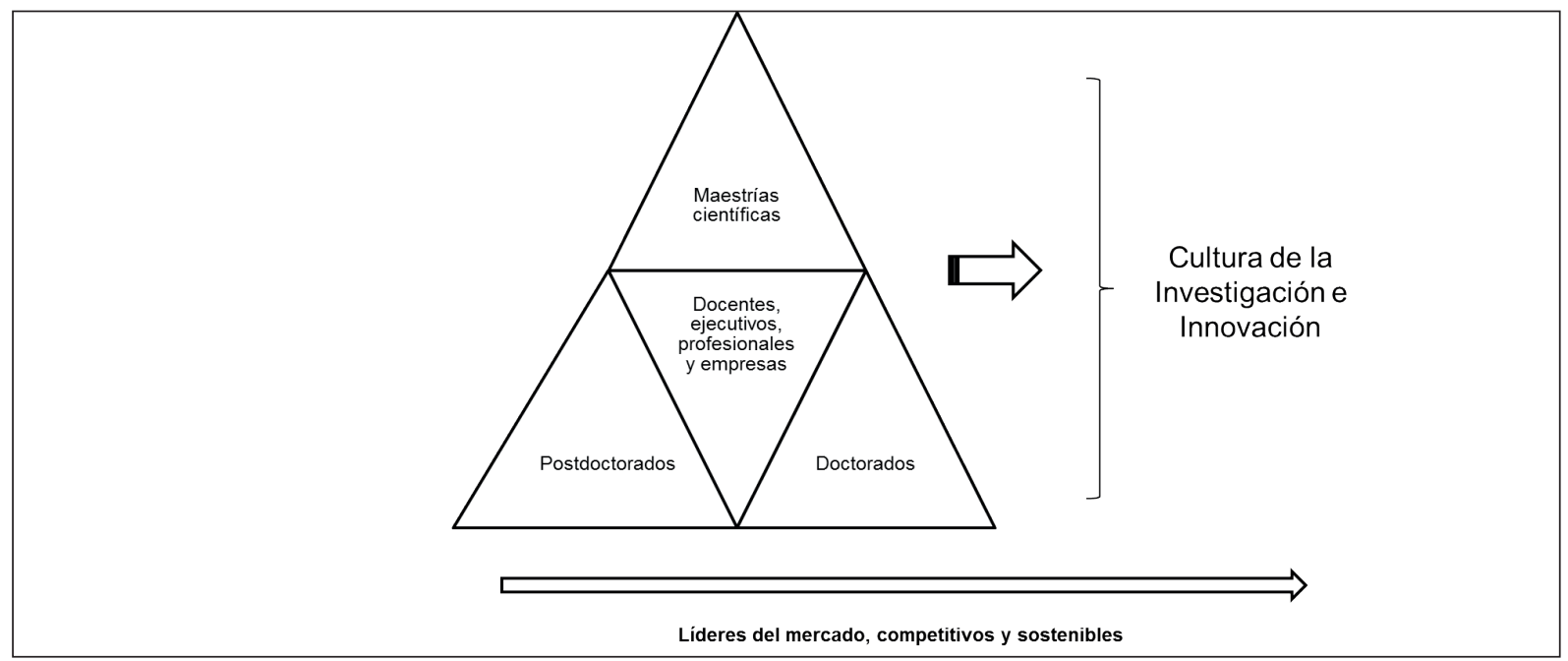

Fuente: Elaboración propia (2016)

Hoy en día, en países desarrollados es muy común ver a un presidente 0 a un joven entre 22 y 27 años con títulos de doctorado porque ellos se han preocupado por estar muy bien capacitados para enfrentar los vaivenes constantes del mercado. El futuro estará más claro con ejecutivos, profesionales o docentes con altos estándares de educación, serán los líderes del mercado, competitivos y sostenibles con altos niveles de crecimiento de sus organizaciones en los mercados.

Los nuevos contextos implican nuevos conocimientos y nuevas normas y disciplinas, y por tanto, la investigación constante en equipo y con comunidades científicas organizadas que operan por fuera del contorno académico, cumplen un factor relevante y significativo para la identificación de lo "nuevo", lo cual si no estamos preparados seremos una nación 0 empresas obsoletas y sin competitividad que se traduce simplemente en ser o no ser exitosos en el escenario global.
Existe una clara relación de desarrollo económico, social y humano con la educación que conlleva a mayor innovación y competitividad por todo el proceso que soporta a los profesionales competitivos, los cuales aportan sustancialmente a las firmas y países.

La buena educación inicia con buenos docentes, preparados integralmente, técnicamente, y por supuesto, muy bien remunerados que enseñen en instituciones educativas de alto nivel con tecnología avanzada y en ambientes propicios para la educación y la innovación. Los docentes deben estar en lo más alto de la sociedad, ellos son la pieza fundamental en el desarrollo económico, social y humano de los pueblos.

Una buena educación desde temprana edad genera para los estudiantes mucha paz espiritual y mental; podemos concluir que la buena educación es sinónimo de avenencia y armonía para los pueblos, esta transforma completamente (Figura 3 ). 


\section{Figura 3.}

\section{Educación y Paz}

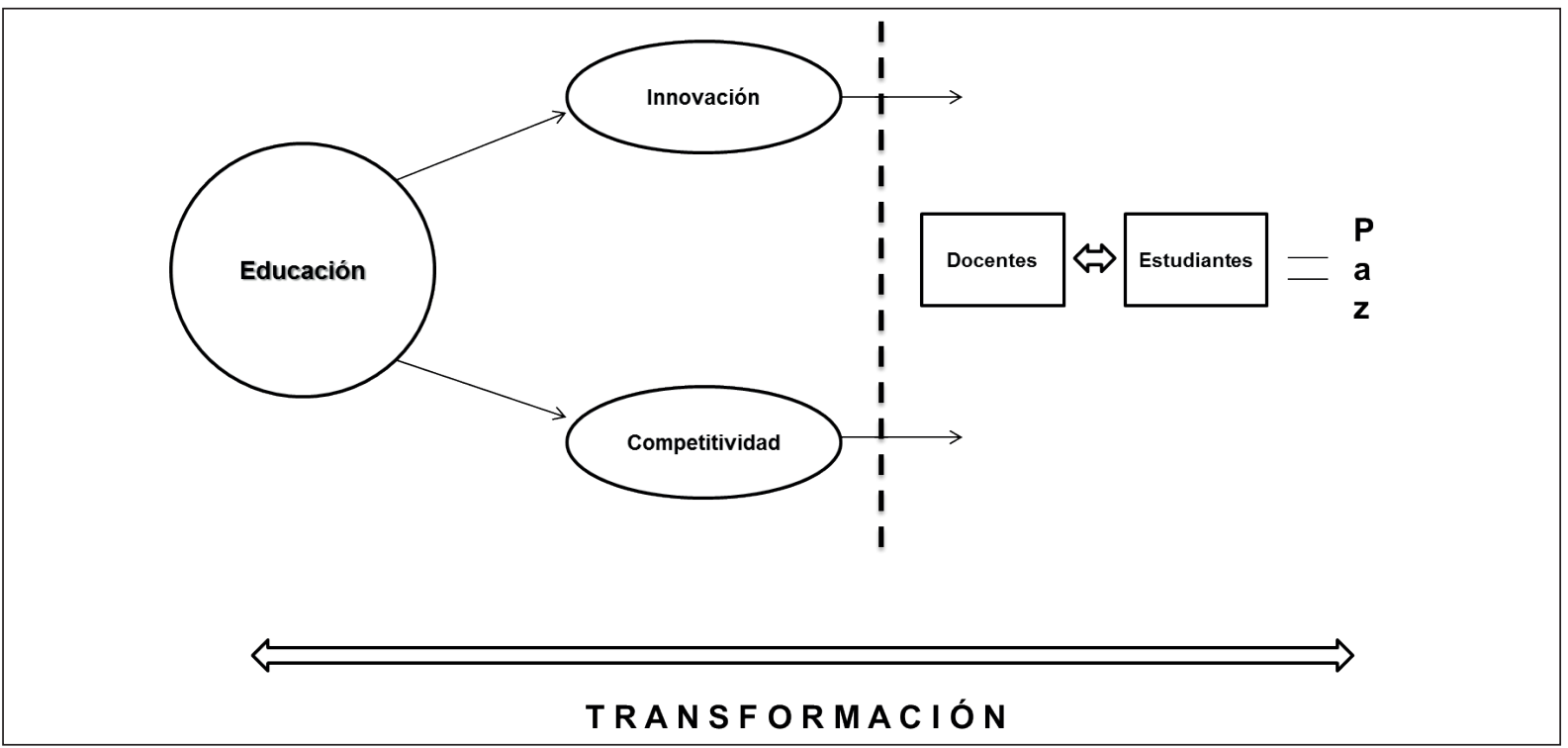

Fuente: Elaboración propia (2016)

\section{Productos innovadores para nuevos consumidores}

Colombia debe incrementar muy duro la inversión en innovación, porque esta representa hoy, un factor decisivo en la negociación mundial. La expedición de patentes ha decrecido notoriamente con relación $a$ años anteriores.

Es necesario ofrecer productos nuevos, con modificaciones que atraigan nuevos compradores y no dedicarnos a hacer copias, muchas veces mal hechas, de otras latitudes. Colombia tiene muchas potencialidades, no podemos depender de dos o tres productos como hace algunos años, debemos buscar otras alternativas e incrementar esas exportaciones no tradicionales.

Hay que expandir nuestro portafolio con productos novedosos y así mantenernos siempre vigentes, pero desde luego, apoyándonos en la creciente tecnología y de esta manera llegar a la innovación. Pero el tema es complejo, pues la relación público-privada debe ser tangible, y todavía existe una gran debilidad en la mayoría de las empresas en cuanto a sus capacidades de innovación.

Mejorar en el mercado depende de los avances de la formación. Acrecentar las capacidades internas, la estructura empresarial, aumentar el talento humano y orientar la cultura organizacional hacia la innovación, es elemental. Para lograr esa competitividad en materia de innovación se necesita tener profesionales aptos, pero también unir esfuerzos entre el empresario, el sector público y la academia, en la unión está la fuerza.

Una debilidad es la ausencia del sostén y financiamiento de las empresas a las universidades para la Investigación y el Desarrollo (I\&D).

Para Moreno-Brid y Ruiz-Nápoles (2009): 
El contexto se confunde más porque en Latinoamérica, como en diversas economías semiindustrializadas, las firmas privadas no cuentan con áreas de Investigación \& Desarrollo y se encaminan a gastar muy poco. Generalmente, cuando requieren tecnología la obtienen de otras latitudes y dedican, en conjunto, muy pocos recursos a la innovación en materia tecnológica, allende a lo que se representa a procesos administrativos o de comercio. Es por ello, que ni los científicos, los investigadores locales son enteramente conocidos como elementos significativos de manufactura en las firmas nacionales, y este tipo de carreras profesionales no constituyen opciones financieras atrayentes para las personas jóvenes (p.21).

El profesor Paul Krugman (1994) afirmó que no son las naciones lo que compiten entre sí, sino las compañías. Para el Banco Interamericano de Desarrollo - BID (2001, p.13) “el desconcierto entre países y firmas puede llevar a creer que la fortaleza económica de una nación se pesa por su superávit comercial externo, que las importaciones no son deseables porque desalojan al empleo nacional, 0 que los bajos salarios de los países son una amenaza para el desarrollo de los países ricos". Es sustancial recordar que un país será competitivo en la medida en que tenga empresas y sectores competitivos y estas empresas desarrollan sus actividades en espacios geográficos concretos (Betancourt, 2014, p.35).

El BID (2001, p.13) manifestó que en "la actualidad la inquietud por esta actividad se ha en las naciones su crecimiento. Sin abandonar las enseñanzas del profesor Krugman. Este fenómeno se está cambiando por una visión corporativa más completa, que contenga la excelencia de lo público sin perder de vista los criterios de las macroeconomía, la disponibilidad financiera, la infraestructura, el capital humano, la innovación y por supuesto la investigación".

¿Compiten entre sí las diferentes naciones? Es probable responder de manera positiva. Los países compiten por firmas que necesitan un lugar, así como por personas inteligentes en busca de trabajo. La discusión notable referente a la competitividad gira alrededor de la correlación que existe en la competencia entre firmas comerciales y las consecuencias que esta trae en diferentes temas relacionados con estas, bien a través de su propiedad o de su ubicación.

En el momento que el entorno de las empresas de un país o región mejora de manera sostenible en cuanto a los niveles de vida, empleos, instituciones educativas de alta calidad, infraestructura y un ambiente notable para realizar negocios que repercute en la atracción de inversiones nacionales e internacionales; el resultado es que las compañías deciden a reunirse dando lugar a la formación de clústers. Para Porter (2003, p.26) “un cluster es la agrupación de firmas o de proveedores, que se dedican a labores similares o complementarias que están relacionados entre ellas por la clase de servicios o productos que manufacturan".

Para Mitxeo, Idigoras y Vicente (2004) los clúster se desarrollan de manera vertical en la cadena de valor donde se incluye a todos los actores del proceso de producción pasando por la industria las compañías, hasta la tecnología como también las instituciones, la academia, los parques tecnológicos y los servicios de información, reciclaje y apoyo técnico.

El cluster es una reunión disímil de empresas diferente a las demás agrupaciones, como son las asociaciones sectoriales (OCDE, 1999; p.85). En la tabla 3 se pueden apreciar claramente sus diferencias. 
Tabla 3.

Diferencias entre clúster y organización sectorial

\begin{tabular}{|l|l|l|l|l} 
& ENFOQUE & $\begin{array}{l}\text { ÁMBITO GEOGRÁFICO } \\
\text { DE ACTUACIÓN }\end{array}$ & \multicolumn{1}{c|}{ OBJETIVO } & \multicolumn{1}{c}{$\begin{array}{c}\text { BASE DE } \\
\text { PERTENENCIA }\end{array}$} \\
\hline $\begin{array}{l}\text { Organización } \\
\text { sectorial }\end{array}$ & Sectorial & $\begin{array}{l}\text { Divisiones } \\
\text { administrativas }\end{array}$ & Representación & $\begin{array}{l}\text { Mismo tipo de } \\
\text { producto y / o } \\
\text { mercado }\end{array}$ \\
\hline \multirow{2}{*}{ Cluster } & Multisectorial & $\begin{array}{l}\text { Resultado del } \\
\text { ámbito de relaciones } \\
\text { interempresariales }\end{array}$ & $\begin{array}{l}\text { Relación entre las } \\
\text { compañías, } \\
\text { identificación } \\
\text { y desarrollo de } \\
\text { actuaciones conjuntas }\end{array}$ & $\begin{array}{l}\text { Visión compartida de } \\
\text { procesos y / o bases } \\
\text { de conocimiento en } \\
\text { una cadena de valor }\end{array}$ \\
\hline & & &
\end{tabular}

Fuente: Ochoa, C. (2002), p.5

La trascendental ventaja de los clúster se emana de su contribución a la mejora de la ventaja competitiva de las organizaciones que lo integran, que favorecería, a su vez, al desarollo de la competitividad de la zona en la que se instala (Mitxeo, Idigoras y Vicente, 2004, p.57).

Entre las tres relevantes direcciones a la mejora de la competitividad para Porter (1999) se citan las siguientes:

- Aumento de la productividad: la cual se ocasiona como resultante de la especialización, de la simultaneidad entre las actividades de las compañías integrantes, de la capacidad de negociación de cada firma que rebaja de manera ostensible los costos de la negociación.

- Fomento de la innovación, derivación de una mejor capacidad para detectar nuevas demandas de los consumidores y otras factibilidades tecnológicas, comerciales o productivas a través de la investigación mancomunada.

- Formación de nuevas firmas, como consecuencia de minimizar el riesgo y los obstáculos de ingreso, conjuntamente con la existencia de nexos preestablecidos y potenciando el número de consumidores que ingresaran a las nacientes empresas (p.219).
Mitxeo, Idigoras y Vicente (2004) afirmó:

A estas variables, se adicionan otros nuevos componentes concernidos en las tipologías del entorno presente, en el cual el conocimiento se ha transformado en un recurso estratégico fundamental. No obstante, la formación siempre será esencial en el progreso de los últimos años donde se registra específicamente su valor respectivo en el desarrollo y la competitividad, subrayando también que su jerarquía es progresiva (p.58).

Entre estos nuevos elementos se destacan: (Mitxeo, Idigoras \& Vicente, 2004)

- El aporte de los clústers a la creación de conocimiento entre las compañías partícipes.

- Los clusters se constituyen así en el asiento para el incremento y desarrollo de bases educativas que permiten optimizar la formación de los trabajadores en todos los niveles de la empresa (p.58).

Es importante mencionar ejemplos de clusters en el mundo como el de Silicon Valey que aloja muchas de las mayores corporaciones de tecnología del mundo como Google, Apple y miles de pequeñas empresas en formación (start-ups), Akamai Technologies, instituida en Boston, es una empresa que provee, 
entre otros servicios, una plataforma de computación distribuida para la entrega de contenidos global de Internet y el reparto de aplicaciones, Paris Saclay en Francia y el cluster minero de Antofagasta en Chile, los complejos en torno a las industrias de computadores en Irlanda y electrónica en Escocia, la industria del calzado en Nuevo Hamburgo (Brasil), de electrónica y programas de computación en Bangalore (India), de instrumentos quirúrgicos simples en Sialkot (Pakistán) y de microelectrónica en Hsinchu Science Park (Provincia de Taiwán), entre otros.

\section{CONCLUSIÓN}

La sinergia que debe existir entre el gobierno, las empresas y las universidades es indispensable, y cada uno de estos actores facilita y apoya la investigación y el desarrollo para llegar a ser innovadores y ser relevantes en el concierto internacional en que estamos inmersos, porque cada día la economía es más global, y las empresas deben estar en dicho escenario o desaparecerán del ámbito de los negocios.

Se recomienda aprovechar todas las oportunidades y los riesgos que conllevan cambios en los procesos de educación e innovación como herramientas importantes para alcanzar niveles altos de competitividad en los países, de lo contrario, sería nefasto y su rumbo sería errático para todas las naciones.

Hoy en día, los nuevos consumidores exigen nuevos productos o servicios especializados a causa de nuevas formas y procesos novedosos muy particulares; las empresas, países y profesionales deben trabajar bajo un solo objetivo y hacer que la educación imperante sea mejor cada día y se convierta en una herramienta crucial de cambio y prosperidad que se traduzca en una competitividad ganadora y transformadora.

La estrategia de competitividad de las naciones debe establecer una orientación con una estrategia de mejora sustentable, tanto a nivel de las compañías como de integración de la cadena productiva a través de una perspectiva de especialización en nichos de mercado y producto en la cadena de valor de las empresas.
La competitividad no está en analizar solo los costos, sino generar un valor adicional a través del desarrollo y entrega de los productos apropiados más rápido y fácil que la competencia.

La nueva estrategia de la competitividad internacional se apuntala en un diseño, en donde la ventaja competitiva recae en la articulación con eficacia operativa y de integración de la cadena de valor. Esta parte desde el diseño de los productos, provisión, producción y distribución al mercadeo. Esto demanda de un programa completo de competitividad global con un enfoque holista aplicado al clúster.

La gerencia moderna debe implantar apropiadamente las ventajas competitivas en sus productos, para que le permitan no solo salvaguardar los mercados ganados, sino que le faciliten el beneficio de otros nuevos. Actualmente, ante la competencia muy ofensiva, es ineludible saber diversificar bien los productos y estar a la vanguardia para aprovechar esas ventajas competitivas que facilitan penetrar en el escenario global en los cuales se presentan muchas oportunidades y amenazas.

Las empresas para lograr ser competitivas deben desarrollar y corregir sistemas propios de su proceso administrativo como son: planeación, organización, dirección y el control con el objetivo de obtener excelentes niveles de satisfacción entre los actores que la circundan, establecidos en una notable base de datos que le ayude a pronosticar y ahondar en los cambios futuros de su contexto. Por ejemplo, ¿Qué productos o servicios demandaran nuestros clientes?, ¿Cómo responderá nuestra compañía? ¿Qué hará la competencia? ¿Somos competitivos? ¿Cuáles serán nuestras acciones para lograr altas tasas de crecimiento? son muchos los cuestionamientos que las compañías deben responder ante su futuro próximo. En todo lo anterior, la educación es la base para tener ventajas de crecimiento ante los nuevos vaivenes del mercado mundial.

Una estrategia profundamente encadenada con la excelencia es la de calidad total como estrategia fundamental de la competitividad. Actualmente, el orbe vive un proceso de cambio rápido y de competitividad internacional en una economía 
muy liberal en la cual es imprescindible un cambio total de orientación en la gerencia de las empresas donde se otorgue más importancia a lo humano como eje fundamental de cambio, y por supuesto, de competitividad.

La innovación es uno de las primordiales variables de competitividad de las organizaciones. Efectivamente, en algunos sectores, la innovación se ha transformado en un componente necesario de conservación. Empero, no obstante para algunas firmas, principalmente para las pequeñas, la innovación es sinónimo de confusión e incompetencia, con la idea que es solo para las grandes compañías, la innovación no es exclusiva, es obligatoria y la educación es la base para lograrla y ser exitosas en los mercados donde desea penetrar.

En suma, con la educación y la innovación de la mano se pueden crear otras maneras para que las regiones del mundo sean más competitivas y desarrolladas.

La innovación y la competitividad se convierten, hoy en día, en factores indispensables en el crecimiento económico de los países teniendo en cuenta que la caída vertiginosa, en el último año de los precios del crudo está poniendo en graves aprietos a los productores de este recurso natural. Así también, muchas materias primas están sufriendo las consecuencias de la deficitaria situación fiscal que se vive en Colombia actualmente.

Colombia no es ajena a esta situación, y por eso, vemos con mucha preocupación, que los recortes presupuestales que el Gobierno está haciendo, obligado por las circunstancias, golpean sensiblemente algunos desarrollos sociales, empresariales y económicos.

Finalmente, imaginemos un estadio ideal de la calidad en la educación e innovación para ser una empresa, región o nación competitiva: (esta historia puede tener parecido con la realidad)

Carlos López reside en un pueblo imaginario donde las oportunidades son prácticamente nulas, debido a que no existen escuelas adecuadas para la enseñanza, no hay una biblioteca, se carece de docentes preparados, existen generadores de pobreza por todo lado.

Un día un líder, la primera autoridad del pueblo, empezó a pensar en la calidad de la educación, invirtió en alta capacitación de los docentes, en infraestructura con tecnología de punta en las escuelas de todos los niveles, educación gratuita y obligatoria, enseñanza de idiomas en los colegios como segunda lengua, creo un centro de excelencia para investigadores, generó una cultura transformadora en la educación.

Los profesores crearon metodologías propias de enseñanza - aprendizaje y les enseñaron a pensar con sentido crítico y desarrollaron sus competencias distintivas.

Al pasar el tiempo el pueblo se fue transformando, se crearon nuevas empresas locales y por supuesto, surgió una gran admiración por cada una de las personas, participantes en este evento, se sentían más felices porque sabían realizar un oficio productivo.

Hoy en día, es un pueblo con personas transformadoras porque están mejor educadas, son innovadoras y cada día que transcurre son más competitivos, no existe techo para ello. Exportan sus ideas y productos debido a que se fueron convirtiendo en los mejores.

Actualmente en este imaginario pueblo la educación es el arma contra la corrupción, pobreza, tristeza, pereza y ceguera ante el progreso.

\section{REFERENCIAS BIBLIOGRÁFICOS}

Araoz, M. (1998). La integración como instrumento para incrementar la competitividad en un mundo globalizado: perspectivas en la Comunidad Andina., CEFIR, Montevideo.

Arrizabalaga, M. (2013a). Así consigue Finlandia ser el número 1 en educación en Europa. ABC. es. Recuperado de http://www.abc.es/20121008/ familia-educacion/abci-consigue-finlandia-numeroeducacion-201210011102.html. 
Arrizabalaga, M. (2013b). Así ha escalado la educación de Corea del Sur al podio mundial. ABC. es. Recuperado de http://www.abc.es/20121020/familia-educacion/abci-escalado-educacion-corea-podio-201210161058. html.

Banco Interamericano de Desarrollo (2001). Competitividad: el motor del crecimiento: Informe de progreso económico y social en América latina. Washington D.C.

Betancourt, B. (2014). Análisis sectorial y competitividad. Ecoe ediciones - Universidad del Valle. Bogotá.

CEIM (2001). La innovación: un factor clave para la competitividad de las empresas. Dirección de Investigación, Comunidad de Madrid.

CEPAL - Naciones Unidas (2004). Desarrollo productivo en economías abiertas. Trigésimo período de sesiones de la CEPAL. San Juan, Puerto Rico

Consejo Privado de Competitividad (2014). Informe Nacional de Competitividad (2014-2015). Recuperado de http://www.compite.com.co/site/ informe-nacional-de-competitividad-2014-2015/

Consejo Privado de Competitividad (2015). Competitividad. Recuperado de http://www.compite.com.co/site/ competitividad/

Comisión Presidencial Norteamericana sobre Competitividad Industrial (1985). Commission on industrial competitiveness report. Washington

Departamento Administrativo Nacional de Estadistica DANE (2015). Archivo nacional de datos. Recuperado de http://www.dane.gov.co/

France. F. (2015). El sistema escolar francés: desde la educación infantil al Instituto. El sitio oficial de Francia. Recuperado de http://www.france.fr/es/ estudiar-en-francia/el-sistema-escolar-frances-desdela-educacion-infantil-al-instituto.html

Garelli, S. (2006). The competitiveness of nations: the fundamentals. IMD World competitiveness Yearbook.

Haguenauer, L. (1989). Competitividade, Conceitos e Medidas. uma Resenha da Bibliografia Recente com Émfase no Caso Brasileiro. IEI/UFRJ, TPD N. 208.

Institute for Management Development - IMD. (2016). Competitividad. Recuperado de https://www.imd.org/

Just, L. (2015). El Sistema educativo Suizo. Recuperado de https://www.justlanded.com/espanol/Suiza/ Guia-Suiza/Educacion/Presentacion

Krugman, P. (1994). Competitiveness: a dangerous obsession. Foreign affairs (special issue), March-April.
León, A. (2007). Qué es la educación. Educere, 11(39), 595-604.

López, A., Méndez, J. \& Dones, M. (2009). Factores clave de la competitividad regional: innovaciones e intangibles, ICE, 848, 125-140.

Mitxeo,J., Idigoras, I., \& Vicente, A. (2004). Los clusters como fuente de competitividad: el caso de la Comunidad Autónoma del País Vasco, Cuadernos de Gestión, 4(1), 55-67.

Moreno-Brid, JC., \& Ruiz-Nápoles, P. (2009). La educación superior y el desarrollo económico en América latina. Serie estudios y perspectivas. Naciones Unidas Cepal. México DF.

OCDE (1999). Managing National Innovation Systems, OCDE, París.

Ochoa, C. (2002). Los clusters y la competitividad en la sociedad de la información y el conocimiento, 4 Foro de Orientación Estratégica, Consorcio Zona Franca de Vigo. Recuperado de http://www.zonafrancavigo.com Oppenheimer, A. (2009). La educación, el secreto de Singapur. La nación.com. Recuperado de http://www.lanacion. com.ar/1166476-la-educacion-el-secreto-de-singapur.

Otero, G. (2006). Competitividad: marco conceptual y análisis sectorial para la provincia de Buenos Aires. Cuadernos de Economía. Ministerio de Economía. Gobierno de la provincia de Buenos Aires. La Plata, Argentina.

Porter, M. (1991). La ventaja competitiva de las naciones. Buenos Aires. Vergara editor.

Porter, M. (2003). Building the Microeconomic Foundations of Prosperity: Findings from the Microeconomic Competitiveness Index. The Global Competitiveness Report: 2002-2003, World Economic Forum, 0xford University Press, Nueva York.

Reinert, E. (1995). El concepto competitividad y sus predecesores: una perspectiva nacional de 500 años. Socialismo y Participación, 72, 21-40.

Romo, D., \& Musik, G. (2005). Sobre el concepto de competitividad. Comercio Exterior, 55(3), 200-215.

Scott, B., \& Lodge, G. (1985). Us Competitiveness In The World Economy. Harvard Business School Press.

Silva, H. (2014). Marketing Internacional: un enfoque práctico. Teoría, casos y talleres. Editorial Alfaomega. Bogotá.

UNESCO (2009). Conferencia mundial de educación superior 2009: las nuevas dinámicas de la educación superior y de la investigación para el cambio social y el desarrollo. Borrador final comunicado. París. 
Universia (2015a). Sistema educativo en Japón. World Economic Forum (2016). Competitividad. Estudios internacionales: estudios en Asia - Pacífico. Recuperado de http://internacional.universia.net/ Recuperado de https://www.weforum.org/ asia-pacifico/japon/sistema-educativo/\#.

Universia. (2015b). Estructura del sistema educativo. Guía de movilidad internacional. Recuperado de http:// estudios-internacionales.universia.net/uk/sistemaWorld Economic Forum (2016). The Global Competitiveness Report 2016-2017. Recuperado de https:// http://www3. weforum.org/docs/GCR2016-2017/05FullReport/ educativo/estructura.html

Waserman, M. (2016). La promoción de lo "novedoso nuevo". Columna de opinión debes leer en: El Tiempo. 4 de marzo, p.15. Bogotá. www.eltiempo.com 